University of Nebraska - Lincoln

DigitalCommons@University of Nebraska - Lincoln

USDA National Wildlife Research Center - Staff Publications
U.S. Department of Agriculture: Animal and Plant Health Inspection Service

2010

\title{
Accounting for Individuals, Uncertainty, and Multiscale Clustering in Core Area Estimation
}

\author{
Ryan R. Wilson \\ Utah State University, ryan.wilson@aggiemail.usu.edu \\ Mevin B. Hooten \\ Utah State University, Mevin.Hooten@colostate.edu \\ Bradley N. Strobel \\ Texas Tech University, bradley.strobel@ttu.edu \\ John Shivik \\ USDA Wildlife Services NWRC, Logan UT, john.shivik@aphis.usda.gov
}

Follow this and additional works at: https://digitalcommons.unl.edu/icwdm_usdanwrc

Wilson, Ryan R.; Hooten, Mevin B.; Strobel, Bradley N.; and Shivik, John, "Accounting for Individuals, Uncertainty, and Multiscale Clustering in Core Area Estimation" (2010). USDA National Wildlife Research Center - Staff Publications. 1284.

https://digitalcommons.unl.edu/icwdm_usdanwrc/1284

This Article is brought to you for free and open access by the U.S. Department of Agriculture: Animal and Plant Health Inspection Service at DigitalCommons@University of Nebraska - Lincoln. It has been accepted for inclusion in USDA National Wildlife Research Center - Staff Publications by an authorized administrator of DigitalCommons@University of Nebraska - Lincoln. 


\title{
Accounting for Individuals, Uncertainty, and Multiscale Clustering in Core Area Estimation
}

\author{
RYAN R. WILSON, ${ }^{1}$ Department of Wildland Resources, Utah State University, Logan, UT 84322, USA \\ MEVIN B. HOOTEN, Department of Mathematics and Statistics, Utah State University, Logan, UT 84322, USA \\ BRADLEY N. STROBEL, Department of Natural Resources Management, Texas Tech University, Lubbock, TX 79409, USA \\ JOHN A. SHIVIK, United States Department of Agriculture, Wildlife Services, National Wildlife Research Center, Utah State University, Logan, \\ UT 84322, USA
}

\begin{abstract}
Core areas are important descriptors of animal space-use patterns, but current estimation methods rely on arbitrary rules and potentially lead to imprecise or erroneous area estimates. We proposed a Bayesian statistical model that incorporates an individual-based method for estimating core area boundaries. The model accounts for boundary uncertainty and multiple scales of clustering by partitioning a home range into $\geq 2$ completely spatially random point patterns defined by a kernel density isopleth. We used data from coyotes (Canis latrans), bobcats (Lynx rufus), and red-shouldered hawks (Buteo lineatus) to estimate core areas for individual animals. We also estimated core areas from simulated point patterns with known boundaries, varying numbers of points, and relative densities of points inside core areas, and compared estimates to those obtained using the $50 \%$ isopleth. Optimal isopleths for the empirical data ranged between $18.7 \%$ and $71.5 \%$. We found no species-specific range of core area isopleths. Across all simulated scenarios, our method outperformed the $50 \%$ isopleth-based estimate, which consistently overestimated core areas. Minta overlap values were $20-40 \%$ higher across all scenarios for our method compared to the $50 \%$ isopleth. Minta overlap values were $>75 \%$ in $90 \%$ of scenarios using our method. Objectively estimating core areas using our individual-based method may lead to improved inference about which behavioral and ecological processes underlie observed space-use patterns because of greater estimate precision.
\end{abstract}

KEY WORDS Bayesian, bobcat, boundary estimation, clustering, core area, coyote, home range, point pattern analysis, redshouldered hawk, space-use patterns.

Landscapes are used and perceived differently by different animals (Haila 2002, Manning et al. 2004). Furthermore, space-use patterns vary not only by species, but by individuals within species, and within individuals during different life history periods (Addicott et al. 1987). Individual specialization and behavioral syndromes could also influence how individuals view and use landscapes (Estes et al. 2003, Sih et al. 2004). Yet, in many aspects of spatial ecology, analytical techniques continue to treat individual animals identically, both within and among species.

Core areas are defined as any area of the home range receiving greater intensity use (i.e., a clustered point pattern; Kaufman 1962, Powell et al. 1997) and are frequently used to answer ecological questions in areas such as interspecific competition, habitat selection, and territorial defense (Neale and Sacks 2001, Chamberlain et al. 2003, Darden and Dabelsteen 2008). Most methods for estimating core areas rely on arbitrary rules that are invariant to intra- and interspecific differences in space-use patterns (Laver and Kelly 2008). Obtaining imprecise core area estimates, or ignoring variation among animals, could affect conclusions drawn in studies relying on core areas as a parameter. Precise spatial analysis is required if we are to infer underlying behavioral processes from observed point patterns (McIntire and Fajardo 2009).

For recent studies employing kernel density estimates (KDE; Worton 1989), 89\% of authors defined a core area by

\footnotetext{
${ }^{1}$ E-mail: ryan.wilson@aggiemail.usu.edu
}

the 50\% density isopleth (Laver and Kelly 2008). Given that different processes underlie space-use patterns for different individuals and species, it is probably not valid to assume that an arbitrary rule will adequately define a core area. Rather, methods used to delineate animal space-use patterns should have a biological underpinning (Shivik and Gese 2000). The arbitrary choice in isopleth is also problematic because it always estimates a core area, even if one does not exist (Fig. 1). It is important that a core area actually be a place of greater intensity use and not a mathematical artifact (Powell et al. 1997).

Many of the features suggested by others as being ideal for core area estimation remain unaddressed. For example, no density-based method is available to objectively choose which isopleth best captures the core area for a given point pattern and a formal definition and ability to account for the internal structure of core areas is lacking (Kenward et al. 2001). Additionally, no core area estimation method exists that accounts for precision of the core area estimate.

Our objective was to increase objectivity in core area estimation by introducing a novel statistical method that addresses many of the features considered ideal when estimating core areas. We used the method and data from bobcats (Lynx rufus), coyotes (Canis latrans), and redshouldered hawks (Buteo lineatus) to show how the method works for real data and the types of inference that can be drawn from more precise core area estimates. We then showed, through simulation, how the model performed under various conditions compared to the 50\% isopleth. 
Random

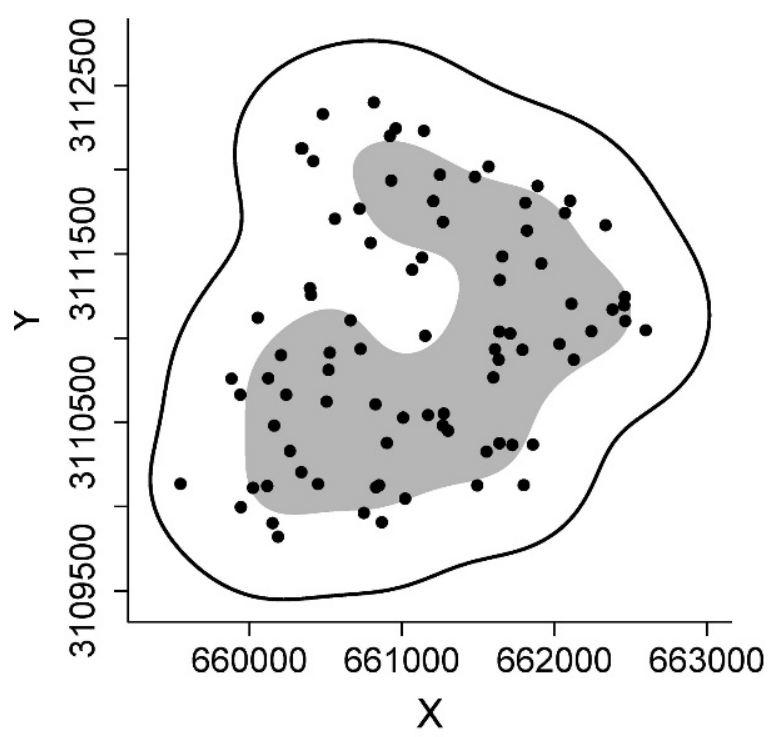

\section{Coyote}

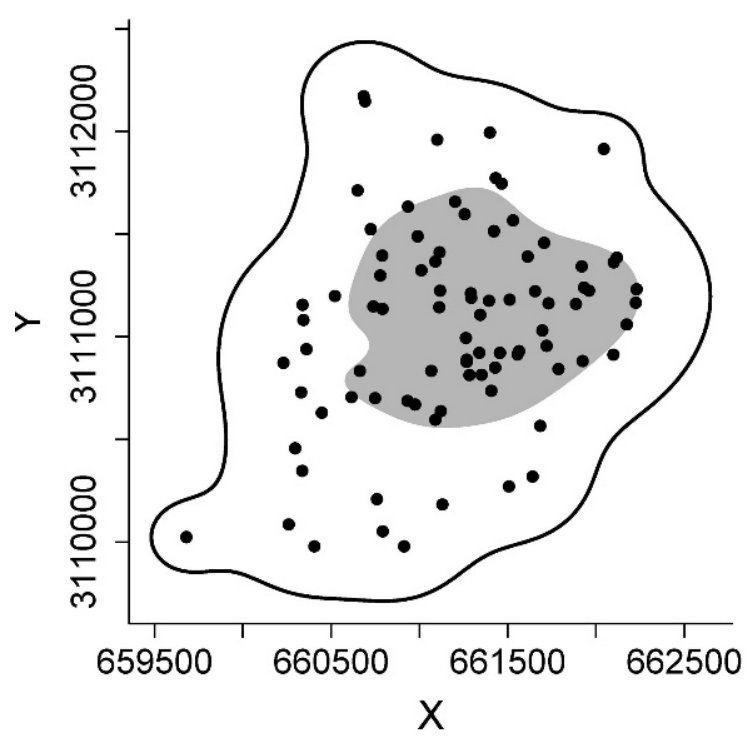

Random

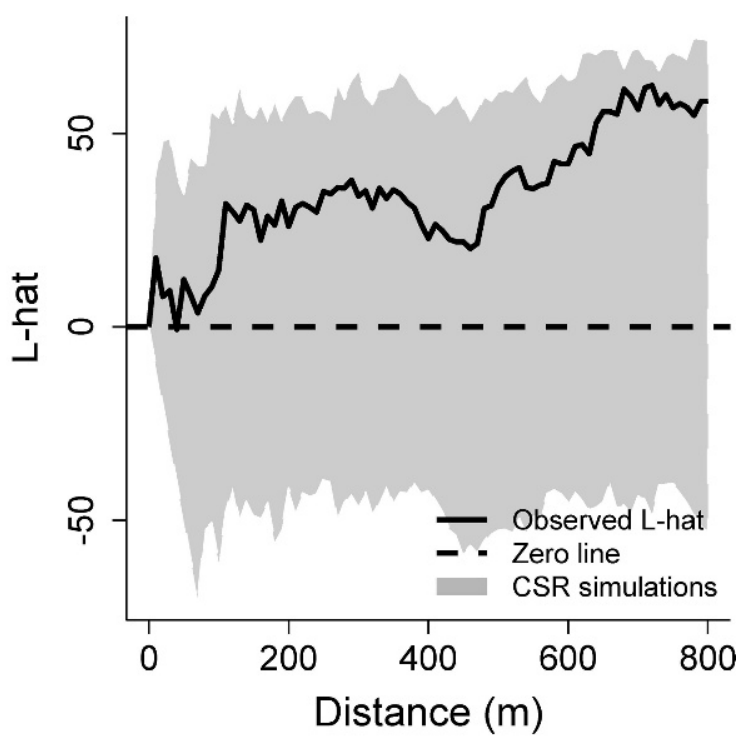

Coyote

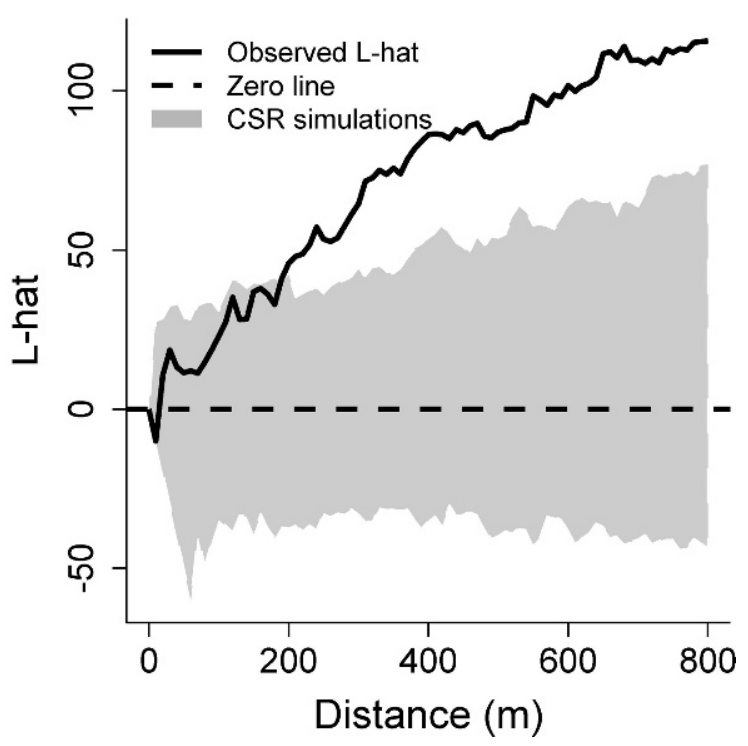

Figure 1. Home range analysis based on 2 separate point patterns: a completely spatial random (CSR) point pattern and a clustered point pattern from a coyote. Outer boundaries for each home range were defined by the $95 \%$ isopleth with fixed-kernel methods using reference bandwidth. Axes for graphs in the left column are based on Universal Transverse Mercator. Both point patterns occur on the same spatial scale and with the same number of points. The shaded area within each home range represents the $50 \%$ isopleth. The L-functions for each point pattern (right column) indicate whether either pattern departs significantly from CSR (as indicated by the observed L-hat rising above the shaded bounds) bounded by their respective home range boundaries (with no boundary correction). The shaded regions for the L-function graphs represents the minimum and maximum L-hat values from 1,000 Monte Carlo simulations of a CSR point pattern on the respective home range boundary. Coyote data were collected from April to October 2008 on the Welder Wildife Foundation Refuge (San Patricio County, TX, USA).

\section{METHODS}

\section{Core Area Model}

The standard definition of a core area indicates that animals behave in such a manner that they occupy regions of their home range with differing intensities (Powell et al. 1997). Therefore, when significant clustering of relocations is indicated in an animal's home range (through a formal hypothesis test using the L-function; Ripley 1976), we assume that a core area exists and that the temporally independent observed point pattern can be partitioned into $v$ separate completely spatially random (CSR) point patterns. We also assume that the partition(s) is random rather than fixed. This assumption can be intuitively justified by considering that animals are likely unaware of some fixed polygon in their home range that denotes core 
space use. It seems unlikely that a definitive core area boundary even exists, especially given that thresholds in resource quality are often indistinct in nature and that boundaries may not be perceived precisely by animals (Powell 2000). Thus core areas might be more appropriately thought of as exhibiting soft boundaries (St-Louis et al. 2004). In the simplest case (i.e., $v=2$ ), animals use core areas and noncore areas with different intensity, but use is uniform within each area, which implies that the observed set of spatial locations should arise from 2 independent multivariate uniform probability distributions with irregular boundaries. We begin by describing the model for the simplest case where $v=2$, then show how the model can be easily modified to account for situations where $v>2$.

The uniform model is difficult to fit; thus, as a discrete approximation, the first step is to transform the relocation data by dividing the home range into a finite number $(m)$ of square grid cells or bins and then summing the number of observed animal locations that fall within each bin resulting in an $m$-dimensional set of counts, $\mathbf{N}$. The appropriate number of bins $(m)$ will vary depending on the total number of animal locations and their distribution within the home range. We found that partitioning the home range into 5002,000 bins worked well in most of the situations we considered. Ideally, the number of bins, $m$, should not influence results, though a sensitivity analysis can verify this if the user wishes to evaluate certain situations. Cell counts, $\mathbf{N}=\left\{N_{1}, \ldots, N_{i}, \ldots, N_{m}\right\}$ represent the number of observed points in each region of the home range. We can then partition $\mathbf{N}$ into core bin counts $\mathbf{N}(C)$ and noncore bin counts $\mathbf{N}\left(C^{\prime}\right.$; where $C$ and $C^{\prime}$ are the core and noncore areas, respectively). If corresponding animal locations are CSR in $C$ and $C^{\prime}$, then we expect the sets of counts to have multinomial distributions with equal multinomial cell probabilities (i.e., $\mathbf{p}_{\boldsymbol{C}}=\left\{1 / m_{C}, \ldots, 1 / m_{C}\right\}$ and $\mathbf{p}_{\boldsymbol{C}^{\prime}}=\{1 /$ $\left.\left.m_{C^{\prime}}, \ldots, 1 / m_{C^{\prime}}\right\}\right)$ in each region, where $m=m_{C}+m_{C^{\prime}}$ are the numbers of bins. Then, if we let $\mathrm{n}_{C}$ and $\mathrm{n}_{C^{\prime}}$ denote the total number of points inside and outside the core area, respectively, we will have the following likelihood:

$\mathbf{N} \mid C \sim \operatorname{Multinomial}\left(\mathrm{n}_{C}, \mathbf{p}_{C}\right) \times \operatorname{Multinomial}\left(\mathrm{n}_{C^{\prime}}, \mathbf{p}_{C^{\prime}}\right)$

Equivalently, we could use a Poisson likelihood with intensities equal to $\mathrm{n}_{C} / m_{C}$, and $\mathrm{n}_{C^{\prime}}, / m_{C^{\prime}}$, though it makes no difference in terms of implementation in this case.

We assume the boundary can be well-described by an isopleth of a $\mathrm{KDE}$ of the observed point pattern. There are other methods for delineating polygons (e.g., convex hull, wombling); however, because $\mathrm{KDE}$ is frequently used in animal space-use studies we employ it here (Laver and Kelly 2008). Any application of the model will also be contingent on the choice of bandwidth parameter, which should be biologically meaningful. In developing a core area model, the $\mathrm{KDE}$ isopleth $(\phi)$ is especially attractive because it is bounded between zero and one and can be treated as a statistical parameter to estimate. Once estimated, $\boldsymbol{\phi}$ completely determines the core area partition $C$. Thus, the likelihood (1) can be conditioned on $\boldsymbol{\phi}$.
We can easily construct a statistical model, using Bayesian methods, that incorporates the likelihood (1) and any prior knowledge about $\phi$ and accommodates uncertainty in the boundary estimate. Here, we only assume that $\phi$ should not be too near zero nor too near one; thus, we specify a vague Beta distribution as a model for $\phi$ with hyperpriors both equal to 1.1. The model is simple to implement as a 1parameter Bayesian model and, although there is nonconjugacy induced through the nonlinearity of $\phi$ in the likelihood, we can address this using an accept-reject-style algorithm such as Metropolis-Hastings (Gelman et al. 2004). In doing so, we seek to find the posterior distribution for $\phi$ given the multinomial count data $\mathbf{N}$ :

$$
\begin{aligned}
{[\boldsymbol{\phi} \mid \mathbf{N}] } & \propto \operatorname{Multinomial}\left(\mathbf{N}_{C} \mid \mathrm{n}_{C}, \mathbf{p}_{C}, \boldsymbol{\phi}\right) \times \\
& \operatorname{Multinomial}\left(\mathbf{N}_{C^{\prime}} \mid \mathrm{n}_{C^{\prime}}, \mathbf{p}_{C^{\prime}}, \boldsymbol{\phi}\right) \times \operatorname{Beta}(\boldsymbol{\phi} \mid 1.1,1.1)
\end{aligned}
$$

where the square bracket notation refers to a conditional probability distribution.

The method we presented thus far is suitable when the data exhibit only 2 distinct partitions of the home range. In cases where multiple scales of clustering occur within the home range, we need to allow for the possibility of multiple scales of core areas. We let these $v$ partitions of the home range be denoted as $C_{1}, C_{2}, \ldots, C_{\mathrm{v}}$. Assuming that each of the sets of points falling within the given optimal partitions are independent and CSR, we can obtain the following posterior distribution using another approximate likelihood in terms of a product of multinomial distributions and $\mathbf{N}$ :

$$
\begin{aligned}
{[\boldsymbol{\phi} \mid \mathbf{N}] } & \propto \operatorname{Multinomial}\left(\mathbf{N}_{C 1} \mid \mathrm{n}_{C 1}, \mathbf{p}_{C 1}, \phi_{1}\right) \times \cdots \\
& \times \operatorname{Multinomial}\left(\mathbf{N}_{C_{v}} \mid \mathrm{n}_{C v}, \mathbf{p}_{C v}, \phi_{v}\right) \times \operatorname{Dirichlet}(\boldsymbol{\phi} \mid \boldsymbol{\alpha})(3)
\end{aligned}
$$

where $\boldsymbol{\phi}=\left(\phi_{1}, \phi_{2}, \ldots, \phi_{v-1}\right)^{\prime}$ is a parameter vector containing the kernel-density isopleth parameters. The natural constraint on the vector $\phi$ is such that each of its elements must fall between zero and one and also sum to one. The Dirichlet model is an excellent probability distribution for this multivariate parameter and, thus, we chose it to serve as a prior distribution for $\boldsymbol{\phi}$. Again we specify a vague prior for the multiscale clustering model by setting the elements of the hyperprior vector $\boldsymbol{\alpha}$ equal to small values (i.e., $\left.\boldsymbol{\alpha}=(0.1,0.1, \ldots, 0.1)^{\prime}\right)$.

\section{Empirical Core Area Analysis}

We captured and fitted very high frequency-transmitters to bobcats $(n=7)$, coyotes $(n=8)$, and red-shouldered hawks $(n=7)$ on the Welder Wildlife Foundation Refuge (WWFR; San Patricio County, TX) between April 2007 and May 2008. We obtained relocations on each individual 4-5 days/week during a 6-month period between April and November 2008. We used triangulation and the maximum likelihood estimator in Program Locate II (Nams 2006) to estimate animal locations. We used $\geq 3$ bearings collected within 20 minutes, between $20^{\circ}$ and $160^{\circ}$ of each other, to estimate locations. We randomly chose start times and animals for telemetry sessions and tested to ensure the data were not autocorrelated (Swihart and Slade 1985). We collected both diurnal and nocturnal locations for bobcats 
and coyotes because they are active during both periods (Neale and Sacks 2001). Data on red-shouldered hawks are limited to diurnal locations to correspond to their primary period of activity (Dykstra et al. 2008).

We defined home ranges by the $95 \%$ density isopleth with reference bandwidth as the smoothing parameter (Venables and Ripley 2002). The 95\% density isopleth produces unbiased and robust home range estimates for species of largely different life histories (Börger et al. 2006); thus, it meets our requirement of having a well-defined home range.

We initially tested each animal's space-use data (using the home range as the spatial domain) for clustering using the L-function (Ripley 1976). For each individual that exhibited clustering in their space-use pattern, we estimated the isopleth that optimally partitioned the home range into 2 CSR regions. We again used the L-function to test each partition for departure from CSR. If there was still evidence of clustering, we estimated the 2 isopleths that optimally partitioned the home range into $3 \mathrm{CSR}$ regions. We continued this iterative procedure until all partitioned regions did not differ from CSR so that our model assumptions were met.

We used R (R Version 2.10.0, <www.cran.r-project.org>, accessed 12 Mar 2010) and functions within libraries splancs (Rowlingson and Diggle 1993), spatstat (Baddeley and Turner 2005), adehabitat (Calenge 2006), MASS (Venables and Ripley 2002), and MCMCpack (Martin and Quinn 2006), for all modeling and analysis. R-code for all analyses and a tutorial are available (<http://www.math.usu.edu/ hooten/other/>, accessed 6 May 2010).

\section{Simulations}

We tested how well the model performed with different sample sizes and relative densities of points between the core area and noncore area with known home range and core boundaries. We constrained simulations by choosing a representative home range and core area boundary from the bobcat data sets. Within these bounded areas we simulated point patterns with 50, 75, 100, 125, and 150 points and with intensities of points inside the core area being 5, 6, 7, 8, 9 , and 10 times as intense as outside the core area. These relative intensities matched the range observed for our empirical estimates. We simulated 10 realizations for each combination of intensity and number of points and estimated the isopleth that best partitioned the home range into 2 regions (i.e., core and noncore). We used the Minta index (Minta 1992) to determine the percent each estimated core area overlapped with the true core area. The Minta index has the ideal feature of accounting for both under and overestimating overlap with the known core area. We also determined the mean percent of points correctly classified as being inside or outside core areas. We determined the Minta overlap values and the percent of points correctly classified using the $50 \%$ isopleth to partition each simulated home range into core and noncore areas.

\section{RESULTS}

We collected a mean (SD) of 85 (27.5), 83 (22.6), and 108 (22.1) locations for each bobcat, coyote, and red-shouldered
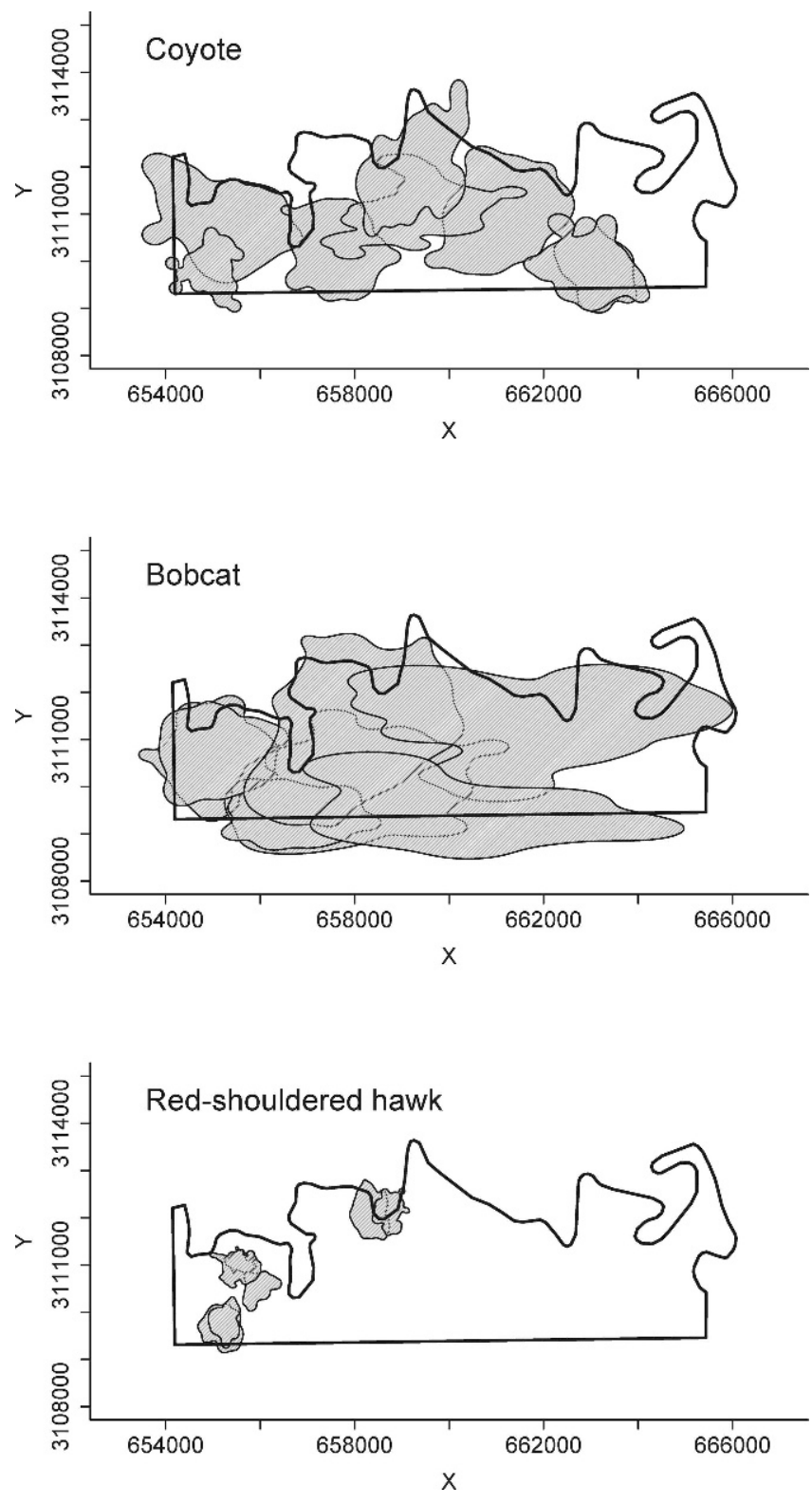

Figure 2. Distribution of home ranges of bobcats, coyotes, and redshouldered hawks (defined by the $95 \%$ fixed-kernel density isopleth using reference bandwidth) on the Welder Wildlife Foundation Refuge (San Patricio County, TX) from April to October 2008. Axes for graphs are based on Universal Transverse Mercator.

hawk, respectively. There was visible interspecific overlap in space-use patterns on WWFR, although home ranges for each species occurred at different scales (Fig. 2). Every individual exhibited clustering in their spatial point patterns, but the scale of clustering differed between species (Fig. 3). Optimal isopleths for delineating the core area ranged between $18.7 \%$ and $71.5 \%$ for individuals across species. There was clear interspecific overlap in the optimal isopleths that delineated the core area with no obvious species-specific pattern (Fig. 4).

Most bobcats (6 of 7) were adequately modeled by partitioning the home range into 2 CSR regions, and only one bobcat required the home range to be partitioned into 3 CSR regions (Fig. 5). Half of the coyotes we studied had 


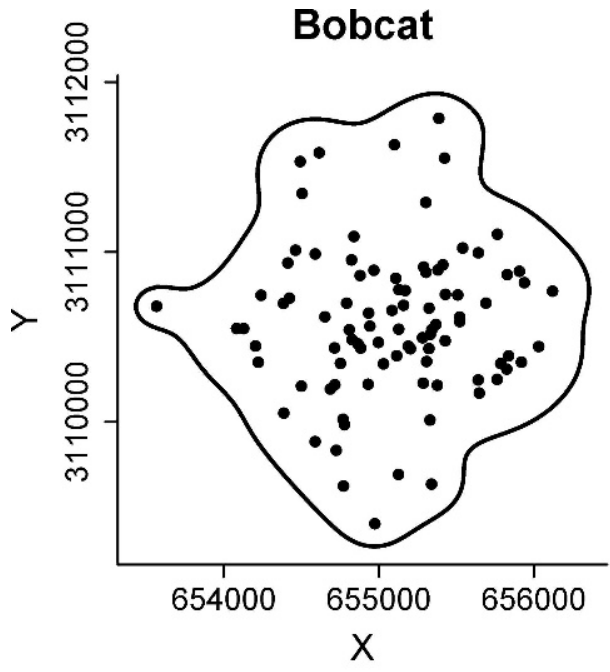

Coyote

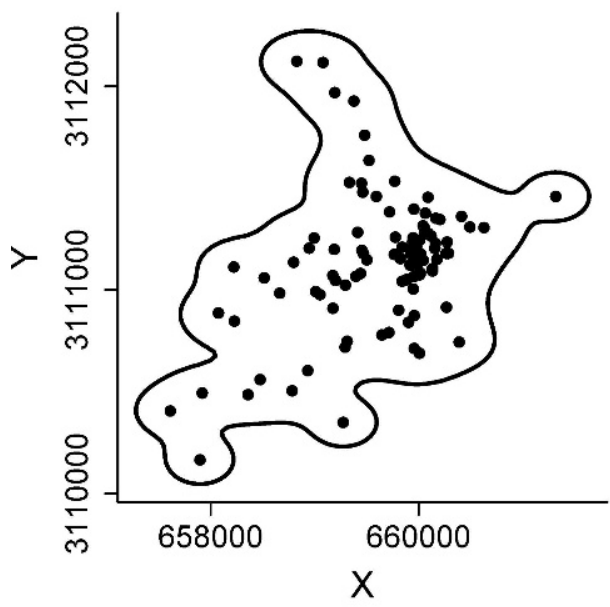

Red-shouldered hawk

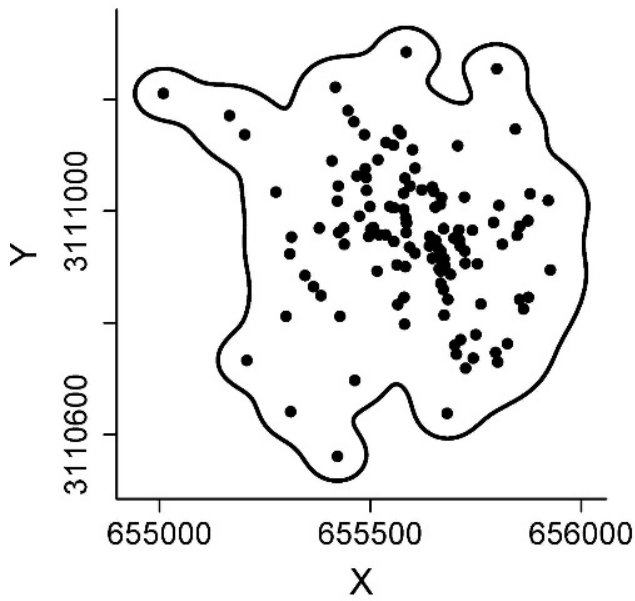

Bobcat

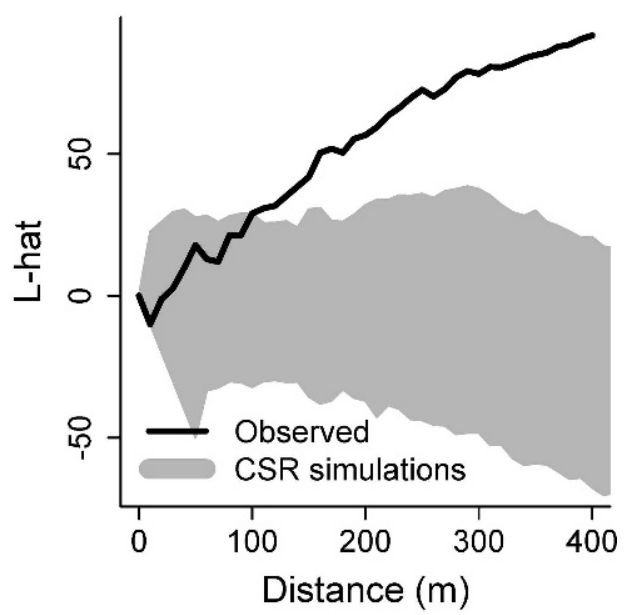

Coyote

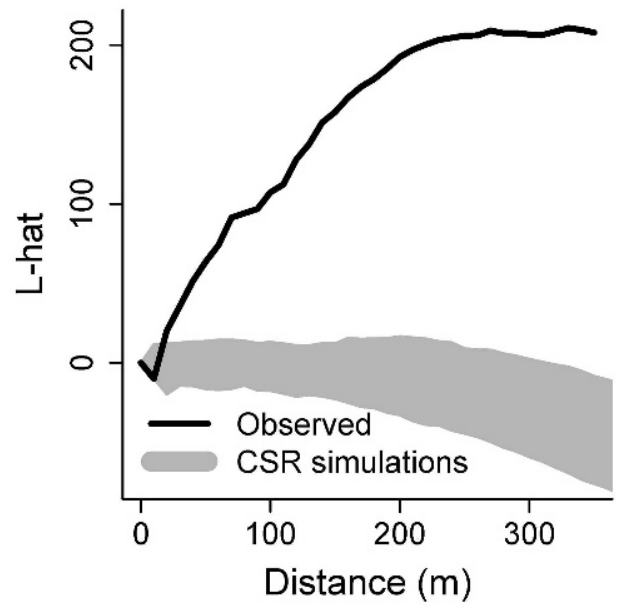

Red-shouldered hawk

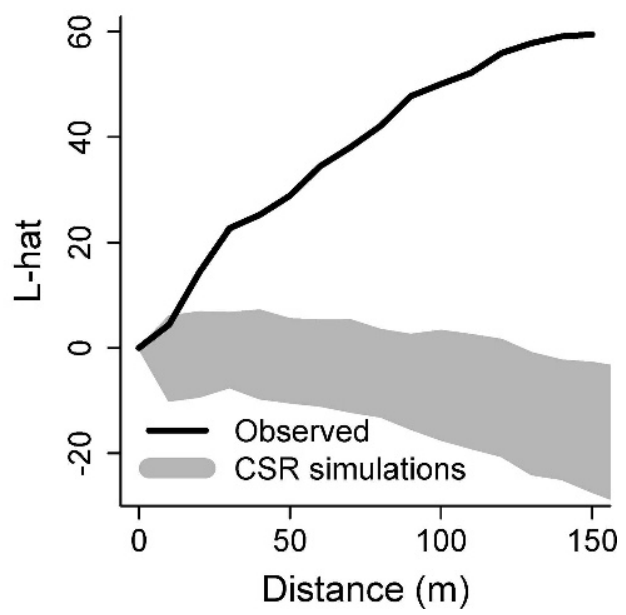

Figure 3. Examples (left column) of data we used for core area analysis obtained from a bobcat, coyote, and red-shouldered hawk on the Welder Wildlife Foundation Refuge (San Patricio County, TX) from April to October 2008. Home range boundaries are defined by the 95\% fixed-kernel density isopleth using reference bandwidth. Axes for graphs in the left column are based on Universal Transverse Mercator. The L-functions for each point pattern (right column) indicate whether each pattern departs significantly from completely spatial random (CSR; as indicated by the observed L-hat rising above the shaded bounds) bounded by their respective home range boundaries (with no boundary correction). The shaded regions for the L-function graphs represent the minimum and maximum L-hat values from 1,000 Monte Carlo simulations of a CSR point pattern on the respective home range boundary. 


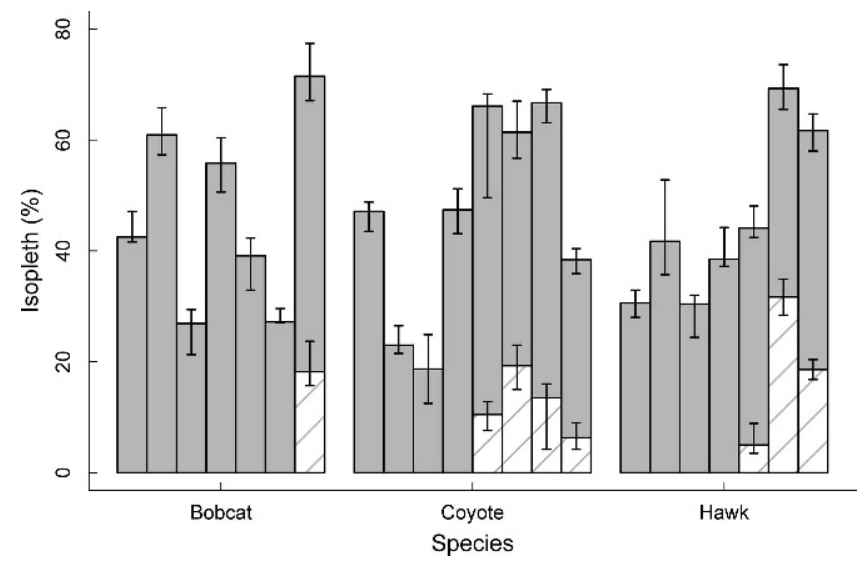

Figure 4. Optimal isopleth values estimated for each bobcat, coyote, and red-shouldered hawk studied on the Welder Wildlife Foundation Refuge (San Patricio County, TX) from April to October 2008. Optimal values are the mode of the posterior distribution of the isopleth parameter $( \pm 95 \%$ credible interval). Solid bars are the optimal isopleths for the outer core area estimates and hashed bars are the optimal isopleths for the inner core area estimates.

home ranges partitioned into 2 CSR regions, whereas the other half required the home range to be partitioned into 3 CSR regions. Four of 7 red-shouldered hawks had home ranges partitioned into 2 CSR regions (Fig. 5), whereas the other 3 were partitioned into 3 CSR regions. Optimal isopleths delineating inner core areas ranged from $5.0 \%$ to 31.7\%, with no apparent interspecific differences (Fig. 4).
There was no systematic difference in the mean number of points within home ranges partitioned into 2 (84.2 [25.9]) and 3 (96.9 [21.28]) CSR regions.

Our method consistently estimated core area boundaries that coincided with the real boundary (Fig. 6) and had high overlap with known core areas in all simulated scenarios (Table 1). The 50\% isopleth always overestimated the core area (Fig. 6) and had Minta index values 20-40\% lower than estimates based on our method in all scenarios (Table 1). Overlap increased with increasing sample size and relative density of points inside of core areas for both methods (Table 1). Across all simulations, the Bayesian method correctly identified $93.5 \%$ (5.2) of locations as being inside or outside core areas, whereas the $50 \%$ isopleth only correctly identified $82.5 \%$ (4.1). The percentage of points correctly classified by the Bayesian method generally increased with increasing numbers of points but not with increasing density of points within the core area (Table 2). The percentage of points correctly classified by the $50 \%$ isopleth increased with increasing numbers of points and with the relative density of points inside the core area (Table 2). In all but 3 scenarios (i.e., points and density combinations), the Bayesian method correctly classified a mean of $>90 \%$ of points, whereas the $50 \%$ isopleth was never able to achieve that level of accuracy (Table 2). The mean isopleth value estimated for the point patterns by the Bayesian method was 21.7\% (7.2).
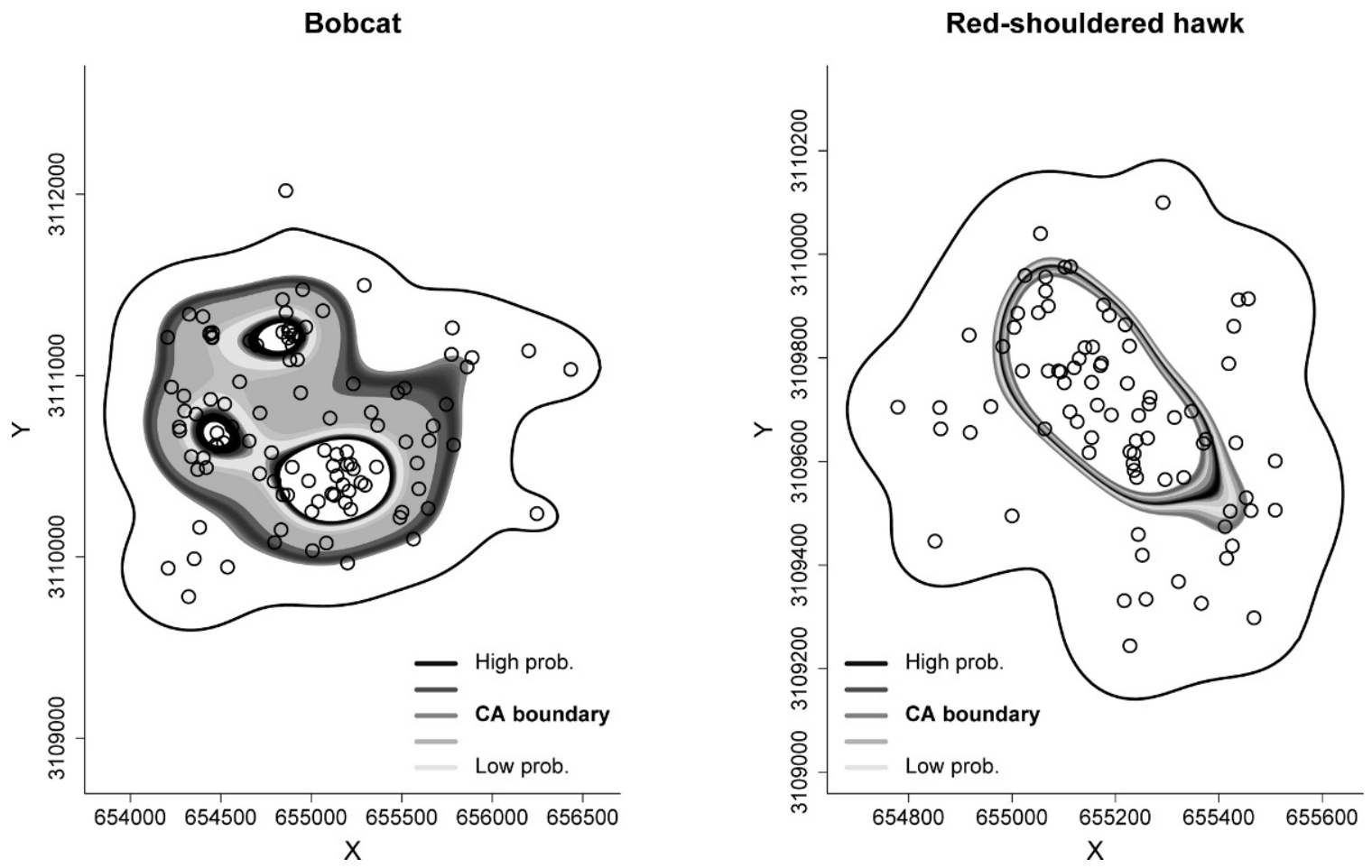

Figure 5. An example of bobcat (left) and red shouldered hawk (right) home ranges on the Welder Wildlife Foundation Refuge (San Patricio County, TX) from April to October 2008 showing uncertainty in the partitioning of home ranges (defined by the 95\% isopleth and reference bandwidth) into core and noncore areas. The bobcat's home range is partitioned into 3 complete spatial random (CSR) point patterns (i.e., multiscale core area model) and the redshouldered hawk's is partitioned into 2 CSR point patterns (i.e., single-scale core-area model). Darker shading of the core area (CA) boundary (or inner core area boundary) indicates those isopleths with the highest probability of delineating the core area. The level of shading is indicated by the posterior distribution of the isopleth parameter. The width of the shaded boundary indicates the level of uncertainty in the estimate. Axes for graphs are based on Universal Transverse Mercator grid projections. 

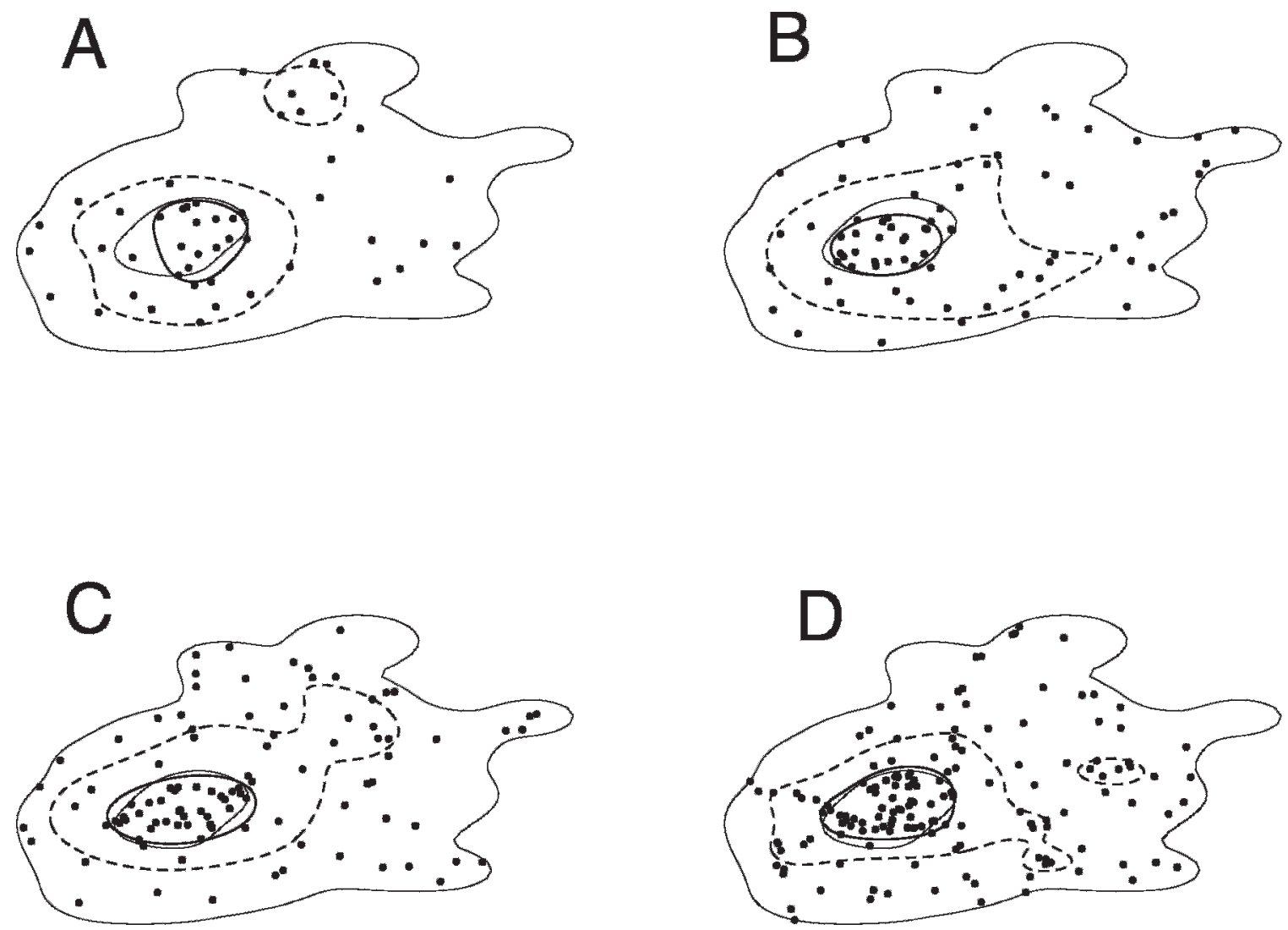

Figure 6. Four realizations of simulated point processes on a realistic home range and core area where density of points are 7 times greater inside core areas than outside. True core area boundaries are denoted as a light solid line, whereas the dark bold solid line and dashed lines represent the core area boundary estimated with the Bayesian method and the $50 \%$ isopleth, respectively. Simulations contained the following number of points and estimated core area isopleths: (A) 50 points, $15.0 \%$ isopleth, (B) 75 points, $13.1 \%$ isopleth, (C) 100 points, $18.9 \%$ isopleth, and (D) 150 points, $22.5 \%$ isopleth.

\section{DISCUSSION}

We presented a new core area estimation method that implements many of the features expressed as ideal by Kenward et al. (2001). Specifically, our model formally accounts for the "inner focal areas" (Kenward et al. 2001:1917), provides an objective kernel-based technique for finding the optimal isopleth for estimating core areas (Kenward et al. 2001:1918), is the first to account for uncertainty in the core area boundary estimate, and allows for the visualization of which segment(s) of the boundary are most uncertain. Other approaches exist for partitioning heterogeneous point patterns into homogeneous regions (Wiegand and Moloney 2004) but do not have the benefit of being implemented in a kernel-density framework or with the benefits provided by Bayesian analysis.

Through simulation, we found that the model performed well in a variety of scenarios and was robust to small sample

Table 1. Overlap between known and estimated core areas based on boundary estimates from Bayesian techniques or the 50\% isopleth. Overlap was measured by Minta index, which varies from 0 (no overlap) to 100 (complete overlap). We used a representative home range and core area boundary estimated from a bobcat data set to simulate points in. We simulated point patterns with 50,75,100,125, and 150 points and with the densities of points inside the core area 5, 6, 7, 8, 9, and 10 times that outside the core area. For each point and density combination, we simulated 10 point patterns, and obtained the mean Minta index value.

\begin{tabular}{|c|c|c|c|c|c|c|c|c|c|c|c|c|c|}
\hline \multirow[b]{3}{*}{ Method } & \multirow[b]{3}{*}{ No. points } & \multicolumn{12}{|c|}{ Density } \\
\hline & & \multicolumn{2}{|c|}{5} & \multicolumn{2}{|c|}{6} & \multicolumn{2}{|c|}{7} & \multicolumn{2}{|c|}{8} & \multicolumn{2}{|c|}{9} & \multicolumn{2}{|c|}{10} \\
\hline & & $\bar{x}$ & SD & $\bar{x}$ & SD & $\bar{x}$ & SD & $\bar{x}$ & SD & $\bar{x}$ & SD & $\bar{x}$ & SD \\
\hline \multirow[t]{5}{*}{ Bayesian } & 50 & 66.4 & 14.2 & 75.0 & 6.7 & 68.4 & 12.6 & 80.1 & 7.2 & 75.4 & 10.2 & 82.0 & 6.5 \\
\hline & 75 & 71.0 & 16.8 & 76.5 & 10.3 & 78.8 & 7.4 & 82.2 & 4.3 & 82.5 & 5.8 & 80.9 & 7.7 \\
\hline & 100 & 79.4 & 3.2 & 81.4 & 7.1 & 84.5 & 5.8 & 83.3 & 3.6 & 82.1 & 7.4 & 85.2 & 5.2 \\
\hline & 125 & 79.4 & 9.7 & 84.7 & 5.3 & 84.0 & 4.8 & 84.9 & 3.2 & 84.2 & 6.3 & 86.7 & 6.1 \\
\hline & 150 & 83.9 & 6.3 & 85.8 & 4.2 & 86.1 & 5.6 & 80.7 & 5.0 & 87.7 & 1.8 & 89.4 & 3.5 \\
\hline \multirow[t]{5}{*}{$50 \%$} & 50 & 41.4 & 2.5 & 45.3 & 3.0 & 47.4 & 5.1 & 48.0 & 2.3 & 53.4 & 4.2 & 52.9 & 4.4 \\
\hline & 75 & 43.1 & 2.4 & 46.8 & 3.4 & 47.6 & 3.2 & 48.7 & 3.0 & 51.5 & 2.8 & 55.8 & 3.9 \\
\hline & 100 & 43.7 & 2.6 & 46.1 & 2.9 & 46.7 & 1.2 & 49.7 & 3.5 & 53.2 & 2.2 & 54.2 & 2.5 \\
\hline & 125 & 43.7 & 0.9 & 45.3 & 1.8 & 48.5 & 2.4 & 49.6 & 2.1 & 52.7 & 1.3 & 55.5 & 3.3 \\
\hline & 150 & 43.8 & 1.3 & 45.9 & 1.1 & 48.2 & 2.6 & 51.0 & 2.7 & 52.3 & 2.2 & 56.0 & 2.5 \\
\hline
\end{tabular}


Table 2. Percentage of points correctly classified as occurring inside or outside of known core areas based on core area boundary estimates from Bayesian techniques or the $50 \%$ isopleth. We used a representative home range and core area boundary estimated from a bobcat data set to simulate points in. We simulated point patterns with $50,75,100,125$, and 150 points and with the densities of points inside the core area $5,6,7,8,9$, and 10 times that outside the core area. For each point and density combination, we simulated 10 point patterns and obtained the average percent of points correctly classified.

\begin{tabular}{|c|c|c|c|c|c|c|c|c|c|c|c|c|c|}
\hline \multirow[b]{3}{*}{ Method } & \multirow[b]{3}{*}{ No. points } & \multicolumn{12}{|c|}{ Density } \\
\hline & & \multicolumn{2}{|c|}{5} & \multicolumn{2}{|c|}{6} & \multicolumn{2}{|c|}{7} & \multicolumn{2}{|c|}{8} & \multicolumn{2}{|c|}{9} & \multicolumn{2}{|c|}{10} \\
\hline & & $\bar{x}$ & SD & $\bar{x}$ & SD & $\bar{x}$ & SD & $\bar{x}$ & SD & $\bar{x}$ & SD & $\bar{x}$ & SD \\
\hline \multirow[t]{5}{*}{ Bayesian } & 50 & 85.7 & 7.3 & 92.9 & 3.8 & 88.4 & 6.7 & 93.1 & 4.8 & 90.4 & 5.0 & 93.9 & 4.8 \\
\hline & 75 & 85.7 & 11.9 & 92.4 & 6.1 & 91.8 & 3.7 & 95.0 & 2.6 & 94.7 & 2.4 & 94.0 & 4.0 \\
\hline & 100 & 94.3 & 2.5 & 93.3 & 3.7 & 95.3 & 3.6 & 95.0 & 2.9 & 95.0 & 4.0 & 93.9 & 4.6 \\
\hline & 125 & 93.3 & 4.6 & 91.0 & 5.8 & 94.5 & 3.9 & 96.1 & 2.6 & 93.0 & 3.4 & 96.2 & 3.8 \\
\hline & 150 & 95.2 & 3.7 & 96.5 & 2.2 & 96.4 & 3.4 & 94.3 & 3.1 & 97.3 & 1.8 & 95.5 & 2.4 \\
\hline \multirow[t]{5}{*}{$50 \%$} & 50 & 74.6 & 2.8 & 77.7 & 3.6 & 75.9 & 3.3 & 82.1 & 2.9 & 82.7 & 2.6 & 83.6 & 2.7 \\
\hline & 75 & 78.2 & 1.7 & 79.6 & 2.3 & 81.6 & 1.3 & 82.0 & 2.7 & 84.3 & 2.2 & 86.7 & 2.0 \\
\hline & 100 & 78.8 & 1.6 & 80.7 & 1.2 & 82.0 & 1.7 & 82.1 & 1.8 & 85.8 & 1.5 & 87.5 & 1.5 \\
\hline & 125 & 79.1 & 1.6 & 80.7 & 0.9 & 83.5 & 1.5 & 84.7 & 1.9 & 86.1 & 2.4 & 88.3 & 1.4 \\
\hline & 150 & 79.5 & 1.4 & 81.9 & 1.7 & 82.6 & 1.8 & 86.0 & 1.6 & 86.4 & 1.6 & 89.2 & 1.2 \\
\hline
\end{tabular}

sizes. The method also always outperformed the 50\% isopleth in estimating the core area. Although accuracy of the $50 \%$ isopleth core area estimate increased with increasing numbers of points and density of points in the core area, this was the result of the optimal isopleth value delineating the simulated point patterns moving closer to $50 \%$. Thus, the only time the $50 \%$ isopleth would be equivalent to the Bayesian estimate would be when the Bayesian estimate is around 50\%, which does not imply that if a researcher has a sufficiently large data set (e.g., $>150$ relocations) that the $50 \%$ isopleth would be just as precise as our approach. Given that the optimal isopleth value delineating the core area is a function of both the number of relocations, the relative density of points inside the core area, and likely the proportion of the home range the core area occupies, it would be unlikely to always converge to $50 \%$. Additionally, these simulations were only of a simple, single-scale core (i.e., 2 CSR processes) example; thus, if multiple scales of clustering are present, the $50 \%$ isopleth will never be able to partition the home range into $>2$ regions. Results of our simulations also show how using arbitrary isopleths to delineate core areas could lead to problems in studies investigating habitat selection due to the large overestimation of core areas.

We used fixed KDE methods to implement our model because of their wide use in home range and core area analysis (Laver and Kelly 2008). Our method is not restricted to one bandwidth selection procedure, definition of home range boundary, or home range estimation methodology. Successful implementation of the model is contingent on the assumption that the home range is welldefined and the chosen home range estimation procedure can adequately characterize the core area. This assumption likely excludes the use of our core area model with the minimum convex polygon method (Hayne 1949) due to the numerous biases and inabilities to accurately estimate animal space-use patterns (Harris et al. 1990). As long as there is a way to link the parameters (i.e., isopleths in our case) to the boundary definition, our model can easily be extended to other home range estimation procedures (e.g., Getz and Wilmers 2004; Horne et al. 2007). Flexibility in home range estimation method is useful because, although $\mathrm{KDE}$ methods perform well in many instances (Börger et al. 2006), in some situations they can lead to biased space-use estimates (Getz and Wilmers 2004), which would also bias the resulting core area characterization.

Relative to application of the model, we suggest that core areas be defined iteratively as we did, working upward from one core area to multiscaled cores incrementally as needed. We recommend checking for additional clustering in each home range partition after fitting the single core model (using the highest posterior mode isopleth as the partitioning polygon). If no additional clustering is evident, then the single core area model results will be used. If additional clustering is evident, then a model with 2 scales (i.e., $v=3$ ) of clustering should be fit. Each of the 3 home range partitions should then be checked for any remaining clustering, at which point the process concludes if none exists. The iterative assumption-checking process also serves as a means of model evaluation. That is, if after fitting the single core area model, evidence of clustering remains in either of the partitioned home range regions, the single core model is inappropriate for the data. Likewise, when all of the clustering tests for the home range partitions indicate no significant deviation from CSR, then the model is appropriately characterizing space use.

In many instances, researchers might only be interested in the core area boundary estimate, and not on the internal structure of the core area, to meet their study objectives. To accurately estimate the core area with our model, however, one would still need to account for the clustering that occurs within the core area, because estimation of the outer core area boundary is based on the optimal partitioning of the home range into CSR processes. Thus, if $3 \mathrm{CSR}$ processes exist, but only 2 are accounted for, the estimated isopleth delineating the core area would be incorrect. Once the 3 CSR processes are accounted for, the isopleth delineating the outer core area boundary can be used.

An advantage of our model is that it can be informed with prior information about the distribution of optimal isopleths for species or social classes to produce more precise estimates. In the application we discussed we used vague 
priors for isopleth values. At present we think there is insufficient information in the literature to inform the model with prior distributions for isopleths given that few studies have objectively estimated isopleths for individuals. More complex models can also be built from our basic model to link other covariates (e.g., environmental, behavioral, temporal) to estimation of core areas.

Although nontrivial in its implementation, our method is intuitive in principle and uses techniques familiar to those studying animal space-use patterns. In addition to being able to accommodate other home range methods, our method can easily be generalized to situations other than analyzing animal space-use patterns (e.g., epidemiology).

\section{Biological Relevance of Core Areas}

Estimates of core areas using our method varied widely between individuals. Similarly, we found no evidence for species-specific rules because there was considerable interspecific overlap in the optimal isopleth estimates. That there would be no species-specific optimal isopleth and such large variation among individuals is expected given the myriad factors affecting animal space use. For example, although subordinate coyotes participate in territorial defense and pup-rearing, they do so at lower levels than dominant individuals (Gese 2001). Thus, we would expect differences in what areas of the home range subordinate and dominant individuals use most intensively, and in the intensity they use those areas, both factors that would affect the optimal isopleth for delineating a core area. If foraging is a dominant behavioral process leading to the formation of a core area, then distribution and density of prey could similarly affect the optimal isopleth for delineating core areas, which is especially true when one considers individual specialization in prey types (Estes et al. 2003). An individual's behavioral syndrome has also been shown to influence its movement patterns (Bremmer-Harrison et al. 2004), thereby potentially affecting the optimal choice in isopleths for delineating a core area.

Describing a home range only in terms of its boundary ignores information about the internal structure of the home range (Horner and Powell 1990). The same can be said of ignoring the internal structure of core areas. Although it is often assumed that a core area is the result of one behavioral process (e.g., foraging), numerous behavioral processes could lead to formation of a core area. Kenward et al. (2001) alluded to the possibility that nonrandom space-use patterns within the core areas might be related to a different behavioral process, which is supported by results from our core area estimates.

Although no male red-shouldered hawks showed evidence for multiscale core areas, all breeding females did (i.e., home range partitioned into $3 \mathrm{CSR}$ regions). An additional female's nest failed early in the breeding season and showed no evidence of a multiscale core area. Breeding season behavior of red-shouldered hawks is sex-specific; breeding females devote more time to incubation and brood-rearing, whereas males forage widely throughout their home range (Dykstra et al. 2008). Thus, core areas for female hawks likely represent a combination of nesting, hunting, and nest defense behavior, whereas male core areas likely represent preferred areas for hunting to provision nestlings. Only one bobcat showed evidence of multiscale core areas, a female with a litter of 4 kittens at a known den site within one of the subcore areas. We analyzed data for another female, but she showed no evidence of multiscale core areas. Given her young age during the study period, however, it is unlikely that she raised a litter of kittens. Patterns in coyote core area analysis are less clear because of unknown social statuses. The number of individuals that exhibited multiscale core areas and the patterns that emerge suggest the life history of an individual, or the ecological neighborhood it currently occupies, might be better predictors of the internal structure of the home range than simply considering the individual's species.

\section{MANAGEMENT IMPLICATIONS}

Regardless of the specific method chosen, based on our findings, we advocate the use of a data-based method for estimating core areas rather than an arbitrary rule, because the latter can lead to misidentification of areas of high use. Increased precision in animal space-use patterns will improve our ability to detect differences among experimental treatments, infer behavioral processes that lead to the formation of core areas, and determine wildlife habitat associations. This can have important implications for setting priorities for conservation and management areas.

\section{ACKNOWLEDGMENTS}

T. L. Blankenship and S. N. Glasscock provided valuable discussion during the development of the manuscript. The staff of the Welder Wildlife Foundation provided support throughout. We thank C. Haralson-Strobel, P. Darrow, S. Meggers, C. Burnett, J. Williams, O. Chan, K. Nelson, N. Cook, and C. Kveton for assistance in data collection. J. Bissonette, W. Link, J. Ver Hoef, and S. Strickler provided valuable comments on earlier versions of the manuscript. Funding was provided by the Welder Wildlife Foundation, American Society of Mammalogists, American Wildlife Conservation Foundation, Houston Safari Club, United States Department of Agriculture Wildlife Services National Wildlife Research Center, and United States Geological Survey. All research was approved by the Utah State University and Texas Tech University Institutional Animal Care and Use Committees. This is Welder Wildlife Foundation contribution no. 689.

\section{LITERATURE CITED}

Addicott, J. F., J. M. Aho, M. F. Antolin, D. K. Padilla, J. S. Richardson, and D. A. Soluk. 1987. Ecological neighborhoods: scaling ecological patterns. Oikos 49:340-346.

Baddeley, A., and R. Turner. 2005. Spatstat: an R package for analyzing spatial point patterns. Journal of Statistical Software 12:1-42.

Börger, L., N. Franconi, G. De Michele, A. Gantz, F. Meschi, A. Manica, S. Lovari, and T. Coulson. 2006. Effects of sampling regime on the mean and variance of home range size estimates. Journal of Animal Ecology 75:1393-1405.

Bremmer-Harrison, S., P. A. Prodohl, and R. W. Elwood. 2004. Behavioural trait assessment as a release criterion: boldness predicts early 
death in a reintroduction programme of captive-bred swift fox (Vulpes velox). Animal Conservation 7:313-320.

Calenge, C. 2006. The package adehabitat for the R software: a tool for the analysis of space and habitat use by animals. Ecological Modeling 197:516-519.

Chamberlain, M. J., L. M. Conner, B. D. Leopold, and K. M. Hodges. 2003. Space use and multi-scale habitat selection of adult raccoon densities in central Mississippi. Journal of Wildlife Management 67:334340.

Darden, S. K., and T. Dabelsteen. 2008. Acoustic territorial signaling in a small, socially monogamous canid. Animal Behaviour 75:905-912.

Dykstra, C. R., J. L. Hays, and S. T. Crocoll. 2008. Red-shouldered hawk (Buteo lineatus). Account 107 in A. Poole, editor. The birds of North America. Cornell Lab of Ornithology, Ithaca, New York, USA.

Estes, J. A., M. L. Riedman, M. M. Staedler, M. T. Tinker, and B. E. Lyon. 2003. Individual variation in prey selection by sea otters: patterns, causes and implications. Journal of Animal Ecology 71:144-155.

Gelman, A. E., J. Carlin, H. Stern, and D. Rubin. 2004. Bayesian data analysis. Second edition. Chapman and Hall/CRC, Boca Raton, Florida, USA.

Gese, E. M. 2001. Territorial defense by coyotes (Canis latrans) in Yellowstone National Park, Wyoming: who, how, where, when, and why. Canadian Journal of Zoology 79:980-987.

Getz, W. M., and C. C. Wilmers. 2004. A local nearest-neighbor convexhull construction of home ranges and utilization distributions. Ecography 27:489-505.

Haila, Y. 2002. A conceptual genealogy of fragmentation research: from island biogeography to landscape ecology. Ecological Applications 12:321-334.

Harris, S., W. J. Cresswell, P. G. Forde, W. J. Trewhella, T. Woollard, and S. Wray. 1990. Home-range analysis using radio-tracking data: a review of problems and techniques particularly as applied to the study of mammals. Mammal Review 20:97-123.

Hayne, D. W. 1949. Calculation of size of home range. Journal of Mammalogy 30:1-18.

Horne, J. S., E. O. Garton, S. M. Krone, and J. S. Lewis. 2007. Analyzing animal movements using Brownian bridges. Ecology 88:2354-2363.

Horner, M. A., and R. A. Powell. 1990. Internal structure of home ranges of black bears and analyses of home-range overlap. Journal of Mammalogy 71:402-410.

Kaufman, J. H. 1962. Ecology and social behavior of the coati, Nasua narica on Barro Colorado Island, Panama. University of California Publications in Zoology 60:95-222.

Kenward, R. E., R. T. Clarke, K. H. Hodder, and S. S. Walls. 2001. Density and linkage estimators of home range: nearest-neighbor clustering defines multinuclear cores. Ecology 82:1905-1920.
Laver, P. N., and M. J. Kelly. 2008. A critical review of home range studies. Journal of Wildlife Management 72:290-298.

Manning, A. D., D. B. Lindenmayer, and H. A. Nix. 2004. Continua and umwelt: novel perspectives on viewing landscapes. Oikos 104:621-628.

Martin, A. D., and K. M. Quinn. 2006. Applied Bayesian inference in R using MCMCpack. R News 6:2-7.

McIntire, E. J. B., and A. Fajardo. 2009. Beyond description: the active and effective way to infer process from spatial patterns. Ecology 90:46-56.

Minta, S. C. 1992. Tests of spatial and temporal interaction among animals. Ecological Applications 2:178-188.

Nams, V. O. 2006. Locate III user's guide. Pacer Computer Software, Tatamagouche, Nova Scotia, Canada.

Neale, J. C. C., and B. N. Sacks. 2001. Resource utilization and interspecific relations of sympatric bobcats and coyotes. Oikos 94:236249.

Powell, R. A. 2000. Animal home ranges and territories and home range estimators. Pages 65-110 in L. Boitani and T. K. Fuller, editors. Research techniques in animal ecology: controversies and consequences. Columbia University Press, Irvington, New York, USA.

Powell, R. A., J. W. Zimmerman, and D. E. Seaman. 1997. Ecology and behaviour of North American black bears: home ranges, habitat, and social organization. Chapman and Hall, London, United Kingdom.

Ripley, B. D. 1976. The second-order analysis of stationary point processes. Journal of Applied Probability 13:255-266.

Rowlingson, B. S., and P. J. Diggle. 1993. SPLANCS: spatial point pattern analysis code in S-Plus. Computers and Geosciences 19:627-655.

Shivik, J. A., and E. M. Gese. 2000. Territorial significance of home range estimators for coyotes. Wildlife Society Bulletin 28:940-946.

Sih, A., A. M. Bell, J. C. Johnson, and R. E. Ziembra. 2004. Behavioral syndromes: an integrative overview. Quarterly Review of Biology 79:241277.

St-Louis, V., M. Fortin, and A. Desrochers. 2004. Spatial association between forest heterogeneity and breeding territory boundaries of two forest songbirds. Landscape Ecology 19:591-601.

Swihart, R. K., and N. A. Slade. 1985. Testing for independence of observations in animal movements. Ecology 66:1176-1184.

Venables, W. N., and B. D. Ripley. 2002. Modern applied statistics with S. Springer, New York, New York, USA.

Wiegand, T., and K. A. Moloney. 2004. Rings, circles, and null-models for point pattern analysis in ecology. Oikos 104:209-229.

Worton, B. J. 1989. Kernel methods for estimating the utilization distribution in home-range studies. Ecology 70:164-168.

Associate Editor: McKelvey. 